\title{
A experiência do Programa Universidade Aberta e suas contribuições para a transformação social
}

\author{
The Open Un iversity Program experience \\ and its contributions to social transformation
}

Débora Cynamon Kligerman 1

Simone Cynamon Cohen 1

Szachna Eliasz Cynamon 1

Cintia Ribei ro da Silva 1

Lilia dos Santos Seabra 2

1 Fundação Oswaldo Cruz, Departamento de Saneamento e Saúde Ambiental, Escola Nacional de Saúde Pública Sérgio Arouca, Fiocruz.

Rua Leopo 1 do Bulhões 1480, Manguinhos, 21041-210,

Rio de Ja neiro RJ.

kliger@ensp.fiocruz.br

2 Uerj/FEBF.
Abstract This paper is a reflection on the Open Un iversity Program that has been developed for twelve years in the Sanitation and Health Department of the Public Health National School/ Fiocruz. The Program is translated as a discussion movement concerning the roots of social inequality, looking for ways to diminish it, through the dialogue between the academic board and the poor local communities. Since its beginning, the Program has motivated the processes of community participation, playing an important role in improving life quality and environment for many poor communities. Usingeduca tion as an instrument of promoting citizenship and community mobilization, it has empowered the communities neglected by government attention, to take proactive actions to maintain their health and dignity. The well-known results obtained by the Open Un iversity Program in Manguinhos, have highlighted its methodology - the research-action-, calling the attention of different public and priva te institutions and NGOs. It has also sensitized the direction of the Public Health National School in order to change it into an official Program of the sch ool, repre sen ting its social commitment to the society. This paper is an analytic repo rt of the Open University, highlighting its path, adopted methodology, main results and evaluation.

Key words Social responsibility Social changes, Social participation, Community development, Citizenship
Resumo Este artigo faz uma reflexão sobre o Pro grama Universidade Aberta, que vem se desenvolvendo há doze anos no Departamen to de Saneamen to e Saúde, da Escola Na cional de Saúde Pública/Fiocruz. O Programa se traduz como um movimento de discussão sob re as raizes da desigualdade social, bu scando caminhos pa ra minorá-la, com a sustentação do diálogo entre meio acadêmico e comunidades desfavo recidas socialmente. Utilizando-seda educação para a cidadania como instrumen to de sensibilização e mobilização comunitária, incentivou a pa rticipação-cidadã, ga ra n tindo-lhes condiçôes necessárias pa ra ações pró-a tivas na conquista e mel ho ria de serviços básicos e essenciais à manu tenção da saúde e dignidade da vida. Os resultados obtidos pelo Universidade Aberta, na minimização dos problemas socioambientais do Complexo de Manguinhos, destacaram a sua metodologia de pesquisa ori entada para a re solução de probl emas - ape squisa-ação. Sensibilizou, ainda, a direção da Escola Nacional de Saúde Pública Sérgio Arouca, constituindo-se um Programa oficial da Escola, representante do compromisso so cial da academia para com a sociedade. O arti go tem como objetivo fazer um rel a to analíti co do Universidade Aberta, destacando sua tra jet ó ri a a metod ol ogia adot ada, seus principais re sultados e avaliação.

Palavras-ch ave Responsabilid ade social, Transformação social, Participação social, De senvolvimento comunitário, Cidadania 


\section{Introdução}

Há pelo menos três décadas a sociedade mundial vem buscando novos rumos para a política social com fins ao engajamento ativo dos social$m$ en te excluídos. Latino-americanos, africanos e membros de países desenvolvidos se impacientam com o con traste existente en tre a excelente qualidade de vida de uns e as situações subumanas de uma grande massa que vive em condições cada vez mais precárias e excluídas do meio social (Ca rdoso, 2004; Jacobi, 2003).

Assim, na condição atual, é oportuna a discussão sobre os caminhos que conduzem a uma socied ade melhor, através de um esforço planejado de redução das desigualdades sociais. Para tanto, con cei tos como os de participação popular, parceria, res ponsabilidade social e empowerment são evocados, adquirindo popularidade cre scen te em programas e projetos sociais em todo o mu n do (Cornwall, 2002).

A participação foi descrita por Cornwall (2002) como um processo pelo qual as pessoas têm papel ativo e influ en te na tom ada de decisões que afetam suas vidas. Demo (1996) definiu a participação como um processo de conquista infindável, um constante vir-a-ser, que não se imagina com pl eta ou suficiente, s eja por parte das comunidades, seja por parte do técnico, do pesquisador, do professor ou do intelectual. A participação não é, portanto, uma dádiva ou con cessão e não admite a tutela por um doador. Ela precisa ser construída, refeita e recriada, necessita entusiasmo e fé nas potencialidades daqu eles que, à primeira vista, parecem excluídos de tu do, exceto da pobreza.

Cornwall (2002) explicou que um desenvolvimen to participativo significa uma parceria con s truída com base no diálogo en tre as várias partes envo lvidas (stakeh old ers). As pessoas deixam de ser simples beneficiadas e tornamse co-responsáveis pelo seu desenvolvimen to. Esta etapa implica mais negociação do que dominação.

O fenômeno da dominação e de sua conseqüência mais direta - a desigualdade social - é um fator históri co com influência sobre a dec isão de participar (Demo, 1996). Peruzzo (1998) observou que, historicamente, o povo brasileiro pos sui pouca tradição de consciência participativa, além da absorção de valores autoritários e da falta de consciência política. Os costumes apontam mais para o autoritarismo do que para o objetivo de assumir o con trole e a co-responsabilidade na solução dos proble- mas. Como produto da dinâmica do autoritarismo, o povo en con tra-se impregn ado de alienação e acomodação.

Demo (1996) acrescenta que o processo históri co de opressão acima referi do deu lugar ao assistencialismo, desfazen do a noção de direi to e cidadania e recrian do a miséria sob forma de tutela. Cardoso (2004) ratifica esta idéia ao com entar que o assistencialismo nada mais é que uma estra tégiade manutenção e fom en to à pobre za, pois cria uma relação de sub serviência e não oferece os meios de superação das carências, ora minorad as.

A inclusão dos indivíduos, como consumidores ou trabalhadores, depende do de senvolvimen to de sua auto - estima, da sua capacidade de comunicação, da sua confiança em seus saberes e da sua capacidade de apren der. É nece ssário, então, uma estratégia metodológica que desenvolva as capacidades latentes do indivíduo, buscando localizar suas potencialidades. Pa ra rom per a inércia e a acom odação é nece ssário, portanto, dispor de alguns instrumentos de participação. Demo (1996) sugeriu a organização da socied ade civil, o planeja m en toparticipativo, a educação para exercício da cidad ania, a cultura como formação histórica da i dentidade comunitária e o processo de conquista de direitos, como possíveis canais de participação, embora não limitados em si. Dentre estes canais, a educação aparece como aquela de função insubstituível, fomentadora da participação e incubadora da cidadania.

A cultura comunitária é outra via que conduz à participação. O lastro cultural próprio que compõe a história de uma comunidade é essencial para o sen ti r-se mem bro de determin ado grupo ou de um projeto concreto de vida. Henriques (1999) destacou a necessidade de que pessoas compartilhem um mesmo imaginário, emoções e conhecimen tos sobre a realidade a sua volta, para gerar reflexão e debate para mudança.

Demo (1996) explicou que a materialização e a organização de uma comunidade dependem de uma série de traços característicos, como língua, mitos, valores, modos próprios de ser e de interagir com a natureza. Uma comunidade só reconhecerá como seu um determi$\mathrm{n}$ ado proj eto se este estiver revestido de traços cultu rais do grupo. Logo, não con si derar a cultura comunitária, em proj etos de cunho social, significa produzir iniciativas imperialistas que não supõem a potencialidade e a criatividade $\mathrm{d}$ a qu ela comunid ade. 
Con forme explicitado por Souza (apud Demo, 1996), os termos educação e cultura são indissolúveis. A educação, via de formação e instrumento de participação, precisa partir das potencialidades do educando e motivá-lo à criatividade própria. A cultura como con tex to da educação é motivação fundamental da mobilização comunitária, essencial para a melhoria da qualidade de vida. Todos os canais de participação convergem para elaborar condições favoráveis de surgimento do cidadão e suas formas de organização.

Há questões sociais que têm gran de po tencial mobil li z ador para a participação comu nitária. As questões relativas ao meio ambi en te parecem ser uma delas. As questões ambientais são chamativas à participação porque convergem, para a sua solução/minimização, di ferentes atores sociais, que, apesar dos interesses múltiplos e de serem afet ados de formas diferen tes pelo mesmo problema, buscam em conjun to resolvê-lo.

A complexa crise ambiental, que ora é enfrentada, remete a uma reflexão sobre os desafios para mudar as formas de pensar e agir, em prol da sustentabilidade socioambiental (Jacobi, 2003). A participação comunitária emerge como a estratégia urgen te e necessária para o enfrentamen to dos muitos problemas decorren tes do descaso com o meio ambien te e socied ades humanas.

Por isso, indepen den temen te dos desafios que se tenha a enfrentar, os proj etos de mobilização social devem prezar pelo exercício da sensibilização sem manipulação, da decisão partilhada, das informaçõesabundantes, da co responsabilidade e representatividade (Peruzzo, 1998), para que não se traduzam em imposição de idéias e retrocesso na con s trução da cidadania.

Em relação aos probl emas socioambientais, há muitas iniciativas privadas que buscam evocar a participação comunitária e a res ponsabilidade do Estado para minorá-los. Estes movimentos, em ergen tes em função da degradação permanente do ambi en te, buscam soluções para problemas sociais que o Estado não consegue solucionar e esti mulam que um maior número de pessoas participem de suas ações e s eus proj etos de preservação / con servação do meio ambiente e de estímulo ao desenvolvimento das comunidades, principalmen te as de s privilegiadas.

Assim, o con ceito de responsabilidade social tem se disseminado na sociedade e no meio corporativo de forma rápida. "A responsabilidade social é uma postura que associa os interesses de todos stakeholders de uma empresa à sua estratégia econômica", com o objetivo de ampliação da ambiência social e da cidadania.

No Brasil, a adoção de uma postura socialmen te re spon sável tem avançado, com a ajuda de entidades que buscam esclarecer o meio corpora tivo e a socied ade da importância dessa iniciativa. Atualmente, $89 \%$ das em presas brasileiras desenvo lvem ações sociais volt adas para a comunidade (Revista Meio Ambiente Industrial, 2004).

Assim, neste con tex to, de st aca-se a iniciativa da Fiocruz desenvolven do o Universidade Aberta, um proj eto social pion ei ro que con tribuiu para minimização dos problemas socioambi entais de uma área em pobrecida da cidade do Rio de Janeiro.

Com mais de 100 anos deexistência, a Fundação Oswaldo Cruz, vinculada ao Ministério da Saúde, tem a missão e o com promisso social de gerar e difundir conhecimen to científico e tecnológi co no campo da saúde, bem como produzir vacinas e medicamen tos de interesse da saúde pública nacional. Portanto, $n$ ada mais natural para uma instituição dedicada ao bem estar social do país do que a atenção dirigida às á reas de maior carência.

De início, o Universidade Aberta representou uma aproximação da instituição com as comunidades do seu entorno. Hoje, representa um exemplo possível de de senvolvimen to local, por meio da comunicação dialógica e da responsabilidade social de um centro de pesquisa de referência internacional, para com as questões e demandas em er gen tes do seu entorno e de construção da participação social.

\section{Históri co do proj eto Un iversidade Aberta}

Em 1993, a Escola Nacional de Saú de Pública Sérgio Arouca (ENSPSA), na figura do profe ssor Szachna Eliasz Cynamon, idealizou e implantou o Projeto Articulado de Melhoria da Qualidade de Vida - Un iversid ade Abert a, integrando as áreas de educação, saúde, habitação e saneamen to ambiental, com intuito de enf rentar os probl emas socioambientais das áreas favel $\mathrm{i}$ adas circ unvizinhas à Fioc ruz.

O proj eto Un iversid ade Aberta era coordenado pelo Departamento de Saneamen to e Sa ú deAmbiental e com pos to por 10 subproj e- 
tos. Contava com a parceria de diversas Unidades e Departamen tos da Fiocruz: Cen tro de Sa úde Escola Germano Si nval de Faria (ENSPSA); Departamento de Ciências Sociais (ENSPSA); Escola Politécnica de Saú de Joa quim Venâncio e, como parcei ros ex ternos, o Comitê de Entid ades Públicas no Combate à Fome e pela Vida (COEP), Coordenação de Pós-Graduação em Engenharia (COPPE/UFRJ) e do Banco do Brasil.

$\mathrm{Na}$ época de implantação do projeto, o cenário das áreas circunvizinhas à Fioc ruz era de profunda degradação socioa mbiental e violência. Ao invés de construir mu ros para pro teger as instalações da Fundação, o professor Cynam on teve o obj etivo de reduzir o distanciamento en tre a Fiocruz e a comunidade do en torn o, contribuindo para que a Fundação desempenhasse seu papel social e as comunidades se sentissem parte dela (Fiocruz, 2003). Mais do que discursar sobre a ética da solidariedade, o professor Cynamon destaca que o mérito do trabalho do pesquisador e do acadêmico não está re s tri to à criação de idéias inovadora $\mathrm{s}$, mas em sua capacidade de canalizar seus estudos pa ra a tra n s formação social (Fiocruz, 2003).

O projeto buscou, então, a proximar o universo acadêmico repres entado por profess ores, pesquisadores e alunos de pós-graduação à re alidade da comunidade do Complexo de Manguinhos, visando à melhoria da qualidade de vida dos moradores, por meio do planejamento e da gestão participativa.

O Proj eto Universid ade Aberta, naqu ela é poca, foi norte ado pela idéia da con s trução da cidadania e ado tou uma metodologia de pesquisa ori en $t$ ada em função da re s o lução de problemas - a metodologia de ensino-pesquisa-ação. Esta vis ava estabelecer um processo de informação contínuo e desbloquear os en traves que existiam no sistema de comunicação, estabel ecendo, assim, uma troca permanen te entre o caminho técnico-científicoe o saber popular.

O processo de troca de conhecimen to en tre os campos do saber - saúde, educação, meio a mbi en te e habitação - foi alimen t ado no interior dos proj etos de pesquisa, gera n do um diálogo, que pode ser traduzido em estratégias e ações para a melhoria da qualidade de vida, re sgatando o valor indivi dual e coletivo da comunidade. Tal metodologia visou, ainda, ao re sga te sistemático da interl ocução en tre proj eto e a comunidade, permitindo a constante avaliação das condições e dos estágios alcançados, f avorecen do a revisão das metas e dos crité rios ado tados através do uso perman en te dos dados de coleta e de sua reutilização nos processos ped a gó gi cos e práticos (Kligerman, 2003). Com isso, pretendeu-se criar condições para que as pró prias comu nidades tivessem auton omia para dar continuidade ao trabalho iniciado e construir novos caminhos para enfrentar os probl emas do seucotidiano.

Thiollent (2002) definiu a pesquisa-ação como um tipo de pesquisa social com base empírica, concebida e realizada em associação com uma ação ou com a resolução de um probl ema coletivo. Nesta metodologia, os pesquisadores e participantes, representativos da situação ou do problema, estão envolvidos de modo coopera tivo ou participa tivo.

A pesquisa-ação, além da participação, supõe uma forma de ação planejada de caráter social e envo lve tanto o saber formal qua n to o in formal, buscan do estabel ecer ou melhorar a e s trutu ra de comunicação en tre os dois universos culturais: o dos especialistas e o dos interessados.

O primei ro passo do Projeto Un iversidade Aberta, em sua fase exploratória, consistiu em descobrir os interessados e suas expectativas e estabelecer um primei ro levantamen to (diagnóstico) da situação, dos problemas prioritários e das eventuais ações. Para tal, foi realizado um reconhecimento das comunidades do Complexo de Manguinhos, com uma pesquisa exploratória que levantou informações sobre os grupos sociais e suas expectativas. Foram realizados, além do perfil socioeconômico, perfil habitacional, avaliação da qualidade da água e monitora m en todo cólera, o levantamento de in dicadores de saúde infantil e da mulher, o levantamento cartográfico demográfico e de ocupação e o diagnóstico da situação de coleta e iden tificação de áreas de risco.

Desemprego, trabalho informal, baixa renda per capita, baixos indicadores de educação e saúde, analfabetismo en tre adu l to s, pre sença da desnutrição, alto índice de gravi dez entre adolescentes, péssimas condições de habitação, saneamen to e ambi en te, ausência de oportunidades cultu rais e de lazer. Es tes foram alguns dos resultados da primeira etapa do Un iversidade Aberta, revelando a ausência ou insuficiência de serviços dirigidos ao atendimento das necessidades essenciais.

O passo seguinte do Universidade Aberta consistiu em apreciar prospectivamen te a viabilidade de uma intervenção de tipo pesquisaação no meio con s i derado. Tra tava-se de detectar apoios, resistências, convergências e diver- 
gências, para fazer um estu do de viabilidade e permitir aos pesquisadores uma tom ada de decisão, a aceitação do desafio da pesquisa sem falsas ex pecta tivas.

Pa ra o de senvo lvimento dessa metodologia, o proj eto Universidade Aberta foi estruturado, inicialmente, com 10 subprojetos que abrangiam ações de monitoramento da saúde, da qualidade da água e da habitação, pesquisas e ações socioeducativas, estratégias de complementação de renda e formação de técnicos em saneamen to e saúde ambiental. Abrangiam, também, ações que pudessem minimizar os probl emas socioa mbientais decorren tes da inexistência de uma política pública adequada ao tratamento dos resíduos sólidos, esgotos sanitários e drenagem do local. Além disso, desenvo lviam pe s quisas para a produção de tecnologias economicamente viáveis nas referidas áreas, utilizando-se de três abordagens: 1) socioeconômica, cultural e educacional; 2) epidemiológica; e 3) ambiental.

$\mathrm{Na}$ abordagem socioeconômica, cultural e educacional, os proj etos eram:

- Un iversid ade Aberta (Palestras socioeducativas): um espaço criado para tra zer as comunidades de Manguinhos às salas da Fiocruz, com a finalidade de promover uma interação pesquisadores-moradores;

- Etn ografia de comunidades em processo de favelização: avaliação antropológica do proce sso saúde-doença;

- Complementação de renda: implantação de atividades com objetivo de geração de emprego; - Formação de técnicos em saneamento e saúde ambi en tal;

- Idearte: prática de atividades técnicas e educativas permeadas por atividades artísticas e culturais, com o objetivo de satisfazer as três premissas básicas para o trabalho em comu nidades pobres: organização social, educação e promoção da auto - es tima.

$\mathrm{Na}$ abord a gem epidemiológica, os proj etos eram:

- De s envo lvi m en to de metodologia para monitoramen to das ações de saúde no âmbito local; - Água e vi gilância sanitária: ca racterização da qualidade da água con sumida em Manguinhos.

$\mathrm{Na}$ abordagem ambi ental, os proj etos s eram:

- Tecnologia apropri ada de saneamen to: água, e sgoto e dren a gem urbana;

- Vi a bilização de um sistema de coleta seletiva e reci cl a gem de lixo em áreas caren tes;

- Investigação de tecnologias alternativas e a propri adas de habitação e urbanização.
Apesar da importância de todos os dez su bprojetos do Un iversid ade Aberta, para o en tendimen to e solução/minimização das questões relevantes no Complexo de Manguinhos, o subproj eto Viabilização de um Sis tema de Coleta Sel etiva e Reciclagem de Lixo em Áreas Caren tes teve mais visibilidade e resultados. Com a redução dos bolsões de lixo e dos problemas deles derivados, pelo emprego de estratégias e ações em educação ambiental, o Un iversidade Aberta chamou a atenção dos órgãos govern amentais e fom en tou a melhoria de atendimento de s te serviço para a população do Com p l exo de Manguinhos.

A participação comunitária no Complexo de Manguinhos, fomentada pelo Universidade Aberta, foi se ampliando a partir da consciência e da tentativa de dar fim aos probl emas socioambientais que afetavam toda a comunidade. Neste mom en to, as melhorias significativas na comunidade, em relação à qualidade de vida e do ambien te foram significativas e forças motrizes para o desenvolvimento dos demais empreendimen tos que o Un iversidade Aberta ainda viriaa realizar.

Associado ao en frentamen to dos probl emas socioambientais, o Universidade Aberta empreen deu esforços e atenção para um dos mais graves problemas sociais que afligiam toda a área: o desemprego. Fez, então, da busca de geração de renda e em prego um forte objetivo a ser alcançado, culminando na criação da Cooperativa dos Trabalhadores Aut ôn omos de Ma nguinhos - a Coo tram.

Composta, em sua maioria, por moradores do Complexo de Manguinhos, a Cootram é prestadora de gran de parte dos serviços de limpeza e con s ervação da Fioc ru z, absorven do cerca de 1.300 cooperados.

Den tro da Fioc ruz e ju n to com a Coo tram, o Universidade Aberta buscou sensibilizar, informar e discutir os principais probl emas ambi entais no campus, estimulan doa apropriação do conhecimen to e mudanças de comporta$\mathrm{m}$ en to dos funcion á rios. Im pla n tou a coleta seletiva de papel, envolvendo funcionários e usuários da instituição. In seriu a participação de quatro adolescentes do Complexo de Manguinhos (en tre 16 a 17 anos) no trabalho da coleta seletiva de papel, sendo estes acompanhados em seu desenvolvimento social e pessoal por profissionais especializados.

O tratamento dado aos resíduos sólidos em toda a Fiocruz e a con tratação da Coo tram redu ziuos ga s too da insti tuição em $38 \%$, além de 
colocá-la em uma posição de vanguarda em relação à responsabilidade social para com os probl emas sociais emergentes.

Diante do su cesso da coleta seletiva de papel, o projeto deu mais um passo e se desdobrou no processo de re a proveitam en to do lixo orgânico, derivado da manutenção dos jardins do campus da Fiocruz, dando início à produção de com pos to vegetal enriquecido e incorpora n do mais qu a tro adolescentes para a realização de stetrabalho. Um ano depois, a procura dos jovens pelo projeto aumen tou consideravelmente. Era comum o atendimen to aos seus responsáveis, desejosos em inserir seus filhos no trabalho, para afastá-los das ruas, das drogas, da marginalidade e aproximá-los da escola e dos cursos de iniciação ao trabalho.

A ajuda de custos oferecida aos adolescentes, provenien te da venda do papel coletado e do com po sto or gâni co produ zi do, foi um grande estímulo dado aos adolescentes, que recebiam cadaqual, em média, um salário mínimo, logo incorporado à renda familiar.

O conjun to das comunidades considerou prioritária a implantação de cursos de iniciação profissional. Estes desejos eram motivados pela grande ociosidade dos adolescentes e adu ltos jovens no Complexo de Manguinhos, com baixos níveis de escolaridade e pouca possibilidade de conquista de emprego. Assim, o Universidade Aberta deu à comunidade do Complexo de Manguinhos a oportunidade de acesso a um el en co de cursos, em condições de viabilizar proj etos indivi duais ou coletivos de geração de renda. Cursos no ensino formal e informal foram, então, ministrados. Destaque deve ser dado aos cursos de jardinagem, construção civil, manutenção de equipamentos, limpeza hospitalar, controle de vetores e compostagem, além das atividades em arte-educação como teatro, dança e música. Tais cursos a proximavam o comu nitá rio dos probl emas locais, e incen tivava à participação na sua solução/minimização.

A grande experiência do Un iversidade Aberta em ministrar cursos comunitários e os resultados do seu trabalho no Complexo de Manguinhos levou o proj eto a de senvolver/ $\mathrm{mi}$ nistrar cursos/aulas em níveis de ex tensão, especialização e mestrado, em que a metodologia de pesquisa-ação era enfatizada, bem como suas po tencialidades e limitações. Dest a que deve ser dado para o curso de Educação para a Gestão Ambiental - o primeiro da cidade do Rio de Janei ro, criado e ministrado pelo Un iversidade
Aberta, preocupado com as questões socioambientais e com a participação comunitária.

Além dos cursos ministrados e da organização da Cooperativa dos Trabalhadores Autônomos de Manguinhos (Cootram), o projeto pre s tou assessorias comunitárias. Pa rticipou da or ganização do Cen tro de Cultura, Arte e Música Pixinguinha (iniciação musical, coral, banda e dança), criando, ainda, cursos de alfabetização e supletivo em 1o e 2 o graus.

\section{Programa Un iversidade Aberta}

Durante a década de 1990, o Centro de Saúde Escola Germano Si nval de Faria (CSEGSF/ENSP) passou por um processo de reorientação de suas ações, potencializandoas ações inters etoriais, já em desenvo lvimento pelo projeto Un iversidade Aberta na área do Complexo de Manguinhos. Estas ações consolidaram, em 1998, uma primeira proposta de de envolvimen to sustentável para o Complexo de Manguinhos - o Desenvolvimento Local, In tegrado e Sustentáved (DLIS). Esta proposta foi firmada por meio do acordo de cooperação técnica entre ENSP/Fiocruz, Associação Canadense de Saúde Pública (CPHA) e a As sociação Brasileira de Saúde Coletiva (Abrasco), com apoio da Agência Ca n adense de Desenvo lvimen to Intern acional (CIDA) (Cynamonet al., 2003).

Pa ra a implantação do DLIS / Manguinhos, con tribuíram o COEP, a Financiadora de Estudos e Proj etos (Finep), a Caixa Econômica Federal, a Companhia de Correios e Telégrafos, o Banco Nacional de Desenvolvimen to Econômico e Social (BNDES) e o Serviços Brasilei ro de Apoio às Micro e Pequena Empresas (Sebrae) e a Prefeitu ra da Gidade do Rio de Jan eiro (PCRJ) (Cynamon et al., 2003).

Para gerir o DLIS / Manguinhos foram c ri ados um Conselho Facilitador, Grupos de Trabalhos Temáticos e uma Secretaria Exec utiva. E ainda quatro grupos temáticos: urbanização; trabalho e renda; saúde e desenvo lvimen to social. Um quinto grupo era form ado pelos coordenadores dos quatro grupos, para con duzir o processo de formulação do DLIS/ Manguinhos.

Através do DLIS/Manguinhos iniciou-se o processo de con dução para a organização de um Fórum de Des envo lvi men toLocal denominado "Acorda Manguinhos". Um grupo foi designado para responder pela elaboração de uma proposta de funcionamento e de mobilização para o Fórum. A partir deste processo, a 
forma de gestão do DLIS/Manguinhos foi repensada, sen do propostos em 2001: o Fórum Comunitário Local, o Fórum Regional de Ma nguinhos e o Conselho de Desenvolvimento e Secretaria Exec utiva, on de foram deliberadas propostas e analisados os meios de execução.

Em 2003, na comem oração de dez anos do Projeto Universidade Aberta, realizou-se um seminário, visando não só à realização de uma avaliação da atuação do projeto, mas também ao estabelecimento de novas parcerias entre a sociedade civil e pública, para o de s envolvimento sustentável no Complexo deManguinhos.

\section{Análise crítica do Proj eto Un iversidade Aberta}

Pa ra a realização desta avaliação crítica do Univers i d ade Aberta foram escolhidos como temas específicos: a relação entre os subprojetos do Un iversidade Aberta; a relação do Un iversidade Aberta com a Cootram; a relação do Un iversidade Aberta com a comunidade; a infra-estrutura de apoio, a divulgação e difusão do Univers i d ade Aberta.

Em relação aos dez subproj etos, percebe-se que, no início do Proj eto Un ivers i dade Aberta, estes estavam interligados, constituindo-se num único de s enho de estra té gias e ações integradas. Ao lon go do tem po, os su bproj etos se distanciaram, devido, por um lado, às dificuldades encon tradas den tro de seus Departamen tos e Unid ades, e por outro, devi do à especificidade de obj etivo de cada um. Assim, à medida que atingiam seus obj etivos, se afastavam do proj eto como um todo. Os su bproj etos remanescentes ganharam identidade com a causa do Universidade Aberta, cria n donovas frentes de atu ação.

Quanto à relação do projeto com a Cootram (como esta se expandiu por todos os setores da Fioc ruz e po s suía rec u rsos para prestação de serviços), sua notori ed ade foi maior que a do a do Proj eto Un iversidade Aberta, que teve parcos recu rsos financei ros até o término do Papes 2 (Programa de Apoio à Pesquisa), suficientes, apenas, para o pagam en to de seus bolsistas. O Un ivers i d ade Aberta não alcançou, assim, a divulgação necessária. Era, constantemente, confundido com a Coo tra $\mathrm{m}$.

A aceitação da proposta do Universidade Aberta não foi tão abrangente quanto se esperava. Pesquis adbres da Fioc ruz ainda têm resistência quan to à aproximação e en trada da comunid ade na Fundação. Ainda hoj e, não há o enten- dimento mais amplo de que o objetivo desse projeto é a melhoria da qualidade de vida e do ambien te do Complexo de Manguinhos; bem como a convivência harm oniosa entre a Fiocruz e a população circunvizinha. Esta postura exi giu que houvesse inovação con st an tepor parte dos pesquisadores envolvidos nos subprojetos, criandoformas de combater esta resistência e de manter viva a proposta inicial do proj eto.

Em relação à comunidade do Com pl exo de Manguinhos, o proj eto teve uma ação mais efetiva, realizando o diagnóstico socioeconômico e possibilita ndoações específicas na área da saúde e do saneamento ambiental, até a formação da Coo tram, em dezem bro de 1994. Em função das dificuldades de intervenção na área e o aumen to da violência, o proj eto vol tou-se para a en trada da comunidade na Fiocruz, realizando cursos e palestras para comunitários. As ações dentro das comu nidades ficaram restritas às associações de moradores, às escolas municipais e à população atendida pelo Centro de Saúde.

Uma análise qualitativa do proj eto aponta $\mathrm{p}$ a ra grandiosidade de sua proposta, a pesar do número reduzido de pesquisadores envo lvidos. Eram tantas ações a serem realizadas que os bo lsistas e pesqui sadores não tiveram tempo e nem a percepção da importância de uma avaliação. Eramrealizados rel a tó rios semestrais, mais de scri tivos e menos avaliativos. Por ocasião do término do Papes 2, a coordenação fez uma proposta de avaliação que não foi aceita e nem contemplada com o financiamen to do Papes 3.

Quanto à infra-estrutura de apoi o, des de a sua criação, o projeto utilizou as instalações dos Departamentos a que pertenciam os pesquisadores envolvidos. A coordenação geral sempre es teve lotada no Departamento de Saneamento e Saúde Ambiental - DSSA/ENSP. Este afastamen to físico foi uma das causas da falta de articulação das ações en tre os subprojetos.

A divu l gação do projeto foi sen do realizada ao longo dos anos, através das articulações do projeto com outros Departamen tos da ENSPSA, Unid ades da Fioc ruz e outros órgãos municipais e estaduais. Com a criação da Escola de Governo em Saúde, coordenação ligada à Direção da ENSPSA, para realização de atividades de ex tensão relacion adas às demandas do SUS, houve uma proposta de divulgação mais efetiva com a or ganização de um site e com a publ icação de com em oração dos dez anos do Proj eto Un ivers i d ade Aberta.

Apesar das iniciativas de divulgação e difusão do projeto, avalia-se o mui to que ainda há 
por fazer para que as experiências do Un ivers i$\mathrm{d}$ ade Aberta possam ser conhecidas e repassadas para outras comunidades com realidade socioambiental semelhante. Atualmente, para a divulgação e a difusão de suas experiências, o Un iversidades Aberta vem sen do apresentando em seminários, fóruns e workshops, visando à democratização do acesso ao conhecimen to acumulado durante os dez anos de estudo, pesquisa e ação sobre desenvolvimento comunitário.

\section{Perspectivas do Programa Universidade Aberta}

A relação da Fioc ruz com o Complexo de Ma nguinhos foi reforçada duran te o seminário de comemoração dos dez anos do Un iversidade Aberta, realizado em 30 e 31 de outubro de 2003. Estavam presen tes vários segmen tos da comunidade acadêmica e da comunidade do en torno, além de representantes de órgãos municipais e estaduais.

Os debates apontaram para o remanejamen to do Proj eto Un iversidade Aberta do Departamento de Saúde Ambiental para a Escola de Governo - ENSPSA/ Fiocruz. E também para a ampliação do Universid ade Aberta de Projeto a Programa. Ao apre sentar o Un iversidade Aberta como um Programa carro - ch efe da Escola de Govern o, a Escola Nacional de Saúde Pública (ENSPSA) busca a requalificação do con ceito de saúde, enten dendo-a como fruto, também, daqualidade de vida e dos ambi en tes. Entende, ainda, que a qualidade de vida e a promoção da saúde são resultados de pactos subjetivos, de definição de direi to e deveres, não somente por parte dos profissionais de saúde, mas pelo con ju n toda sociedade. Há o en tendimento da necessidade de participação dos vários cam pos do saber; apontan do para a interdisciplinaridade, a transdisciplinaridade e para a prática da intersetorialidade.

Estes princípios e estratégias constituem a base de formação do Programa Un iversidade Aberta, que está calcado no tripé: Participação - como busca da autonomia dos indivíduos, dos grupos sociais e da eqüidade social; Responsabilidade Social, como dever de uma instituição que aplica o seu saber cien tífi cona con strução de uma sociedade mais digna e justa; e In ters etorialidade, porque a participação, para ser efetiva, deve envo lver a socied ade civil e os vários campos do saber (Ca rvalho, 2003).
Neste contexto, o seminário de com em oração dos dez anos do Projeto Universidade Aberta elegeu, para continuidade de atuação do Programa Un ivers i d ade Aberta, quatro grandes temas: Ambientes Saudáveis; Geração de Trabalho e Renda; Educação para o De senvolvimento; e Sociedade e Cultural. El egeu, ainda, como áreas de atuação: Resíduos Sólidos; Habitação; Recursos Hídricos; Alimentação; Capacitação e Informação. Cada área de atuação fez interf aces com as questões referen tes à qu alidade do ambiente, à geração de emprego e renda, à educação e à sociocultura local, conforme apre sen tado no qu ad ro 1.

No momen to, o Programa Universidade Aberta se expandiupara outros municípios da Baixada Fluminense, ex portan do sua ex periência e soman donovas forças, que possam apoiar e dar continuidade a sua maior causa: a promoção da qualidack de vida pela associação do saber acadêmico.

\section{Con clusão}

Neste artigo foi feita uma análise crítica sobre o Programa Universidade Aberta, mostrando a evolução do projeto a programa, culminando em um movimento de transformação social que discute as raízes da desigualdade social e formas de minorá-la.

Inicialmente, reconhecendo a necessidade de uma sociedade mais justa, o proj eto foi elaborado embas ado na solidariedade, na ampliação da cidadania e no entendimen to da biodiversidade e da eqüidade como princípios fundamentais para garantia da sobrevivência da espécie humana. Afinal, a saúde de um é indispen sá vel para a saúde de todos.

Desde o início de sua atuação, o Un iversi$\mathrm{d}$ ade Aberta bu scou trabalhar em prol do agir coletivo, conjunto e participativo, fundamentando as bases para uma organização social forte e solidária, que ga ran tisse a expressão das minorias, efetivan do seus direitos e fortalecendo a integração da diversi d ade e do conjunto.

A formação da coopera tiva repre sen tou um grande passo em direção à melhoria da qualid ade de vidaemManguinhos. Quan do foi criada, em 1994, a Coo tram tinha 200 cooperados. Atualmente conta com 1.300 cooperados, representando o aproveitamen to de vinte por cen to da força ativa de trabalho da região.

Com o diagnóstico foram leva $\mathrm{n}$ adas as habilidades dos moradores, que foram divididas 


\section{Quadro 1}

Áreas de Atuação do Un iversidade Aberta.

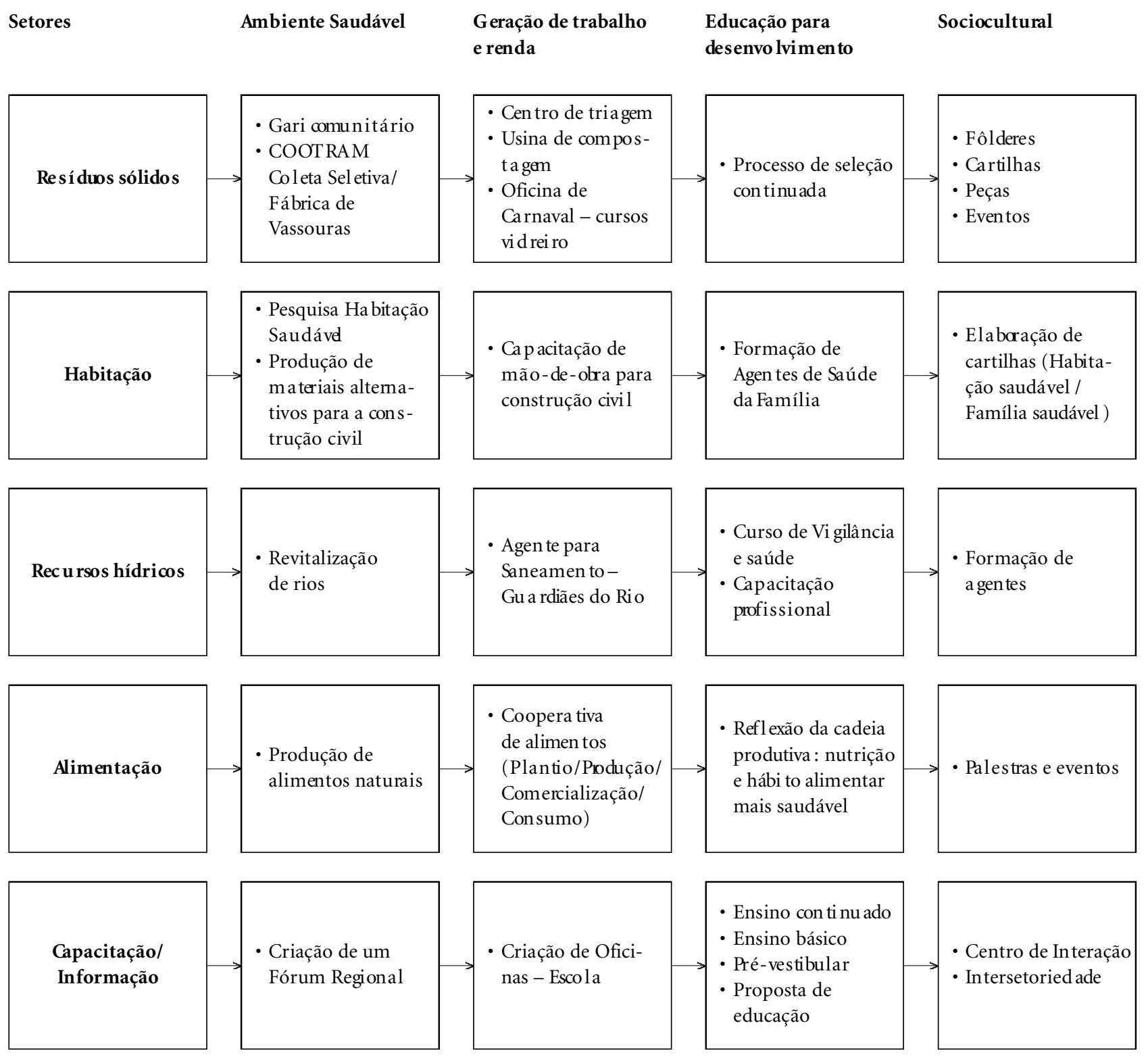

em setores específicos da Cootram como: jardinagem / compostagem e col eta sel etiva (retirando os meninos das ruas e lhes oferecendo cu rsos e ativid ades); s erviços gerais; ma nutenção civil e de equipamentos; controle de vetores; higi enização de biblio tecas; fábrica de tij olos; fábrica de corte e costura; fábrica de fraldas descartá veis; alimentação, administração e recursos humanos (realização de palestras semanais com tema escolhido pelos cooperados); centro cultural Pixinguinha (orquestra infan- to - juvenil, coral, dança afro-brasileira e capoeira) e telecurso 1o e 2o grau (para alfabetização de adultos).

Existem hoje mais de mil pessoas que estão na fila de espera para entra rem para a Cootram. Com a geração de trabalho e renda, ocorreu um processo de amadu recimen to coletivo das comunidades que compunham o Complexo de Manguinhos. Foi criado um Conselho Administra tivo e fiscal na coopera tiva com po sto por representantes das comunidades e pes- 
quisadores da Fundação Oswaldo Cruz. As associações se or ganizam comunita ria mente, elegem priorid ades e lutam junto aos órgãos competen tes pela melhoria das condições de vida em Manguinhos.

A experiência de intervenção social protagonizada por atores sociais das comunidades da Fiocruz e de Manguinhos, através do Programa Un iversidade Aberta, foi bem-sucedida, estandoevi dentes os princípios norte adores da m obilização e participação social.

Com o trabalho de coleta seletiva e compostagem propo s to, foi possível garantir con dições para a melhoria da qualidade de vida dos adolescen tes envolvidos no projeto, oferecendo-lhes oportunidades de crescimen to intel ectual, afetivo e psicológico, asseguran do-lhes o re s pei to e a garantia dos seus direi tos estabel ecidos por lei, e prom ovendo o fortalecimen to de seus laços sociais e comunitários.

O projeto Universidade Aberta propiciou aos seus participantes mais do que informação. Criou uma interação própria entre seus públicos, fortalecen do o vínculo com o proj eto para capacitá-los a tomar iniciativas espontâneas e con tri buir com a causa den tro de suas especialidades e possibilidades. Mais uma vez, a Fiocruz apres en tou sua condição de cen tro de referência em bem-estar social e qualidade am- bi ental, consolidando a con strução da cidadania na socied ade.

Enfim, acredita-se que, com a realização de um trabalho participativo que permite a troca constante entre o saber técnico-científico e o saber popular, foi conseguida a aproximação efetiva da academia com a comunidade, promoven do a sensibilização, mobilização e or ganização comunitária. Foi possívd ga ran tir passos progressivos para o processo de melhoria da qualidade de vida da população moradora do Complexo de Manguinhos e envolvida no projeto. E ainda oferecer oportunidades de crescimen to intelectual, a fetivo e psicológi co, assegurando-lhes o respeito e a garantia dos seus direi tos estabel eci dos por lei e prom ovendo o fortalecimen to de seus laços sociais e comunitários. Embasado numa proposta transform adora, o trabalho primou pelos princípios da participação, solidariedade, disciplina, ética e ju s tiça social.

O próximo desafio do Programa Un iversidade Aberta é a sua transmutação em uma Coordenação da Direção ENSPSA que articule os proj etos sociais, $\mathrm{s}$ eus pesquisadores e população. Tudo isto dentro de um movimen to de transformação social da ENSPSA, como uma extensão da Academia, na busca de caminhos que diminuam as desigualdades sociais.

\section{Colaboradores}




\section{Referências bibliográficas}

Ca rdoso R 2004. Sustentabilidade, o desafio das polític a s s ociais no século 21. São Paulo em Perspectiva 18 (2): 42-48.

Carvalho AI 2003. Intersetorialidade na construção de saúde - a reforma sanitária como reforma social. (Manuscrito).

Cornwall A 2002. Beneficiary, consumer, citizen: perspectives on participa tion for poverty reduction. Sida Stu dies. N. 2, Sweden .

Gynamon SE, Kligerman DC, Gomes AF, Seabra LS \& San tos N 2003. Universidade Aberta: uma con tribuição para a Gestão Ambiental Su s tentável, pp. 79-98. In L Zancan, R Bodstein E WB Ma rcondes (orgs.) 2004. Promoção da saúde como caminho pa ra o desenvolvimen to local: a experiência em Manguinhos - RJ. Abrasco-CIDA-CPHA-FINEP-Fiocruz, Rio de Janeiro.

Demo P 1996. Participação é conquista. Cortez, São Paulo.

Fiocruz 2003. Dez anos de Universidade Aberta. O encontro da Fiocruz com as comunidades de Manguinhos pela tran sformação social. Fiocruz/ENSP/Escola de Governo, Rio de Jan ei ro.
Henriques MS, Braga CS \& Mafra RLM 1999. Planejamen to da co municação pa ra a mobilização so cial: em busca da co - responsabilidade. Dis ponível em <http:// wwwportal-rp.com.br $>$. Acesso em 3/mai/2005.

Jacobi P 2003. E ducação ambiental, cidadania e su stentabilidade. Cadernos de Pesquisa 118:189-205.

Kligerman, DC 2003. Projeto Universidade Aberta - Contribuição de dez anos para a Gestão Sustentável do Complexo de Manguinhos, pp. 10. Anais do 22o Congresso de Engenharia Sanitária e Ambiental, Joinville.

Peruzzo CMK 1998. Comunicação nos movimen tos populares. Vozes, Petrópolis.

Revista Meio Ambiente Industrial. Ser socialmente responsável é um diferencial de mercado. Ano IX, ed. 51, set/out, 2004

Thioll ent M 2002. Metodologia da pesquisa-açãa Cortez, São Paulo.

Artigo apresentado em 6/05/2005

Aprovado em 9/06/2005

Versão final apresentada em 4/07/2005 